\title{
Efficacy of 694-nm Fractional Toning Ruby Laser in the Treatment of Malar Melasma
}

\author{
Jun Hyun Kim \\ Eun Soo Park \\ Seung Min Nam \\ Han Gyu Cha
}

Department of Plastic and Reconstructive Surgery, Soonchunhyang University Bucheon Hospital, Bucheon, Korea
Received December 8, 2020

Accepted February 8, 2021

\footnotetext{
Correspondence

Eun Soo Park

Department of Plastic and Reconstructive Surgery, Soonchunhyang University Bucheon Hospital, 170 Jomaru-ro, Bucheon 14584, Korea Tel.: +82-32-621-5319

Fax: +82-32-621-5016

E-mail: peunsoodsschmc.ac.kr

(C) Korean Society for Laser Medicine and Surgery

(c) This is an open access article distributed under the terms of the Creative Commons Attribution NonCommercial License (http://creativecommons.org/ licenses/by-nc/4.0) which permits unrestricted noncommercial use, distribution, and reproduction in any medium, provided the original work is properly cited.
}

\author{
Background and Objectives \\ Among the numerous available therapies, the usefulness of laser \\ treatment has been proven in melasma, especially for refractory to \\ topical agents or chemical peels. This study evaluated the effectiveness \\ of 694-nm wavelength fractional toning ruby laser in melasma \\ presenting on the malar area.
}

\section{Materials and Methods}

This was a retrospective study of 40 melasma patients treated with 694$\mathrm{nm}$ wavelength ruby fractional toning laser for a total of 8 cycles at intervals of 2 to 3 weeks. Two independent investigators assessed the photographic findings and evaluated the severity of melasma by applying the Melasma Severity Scale (MSS). Subject satisfaction score was also surveyed at every visit.

\section{Results}

After the 4th treatment, subjects were continuously satisfied with their results. At the 8th visit and 1-month after the last treatment, MSS was determined to be significantly improved as compared to the first visit. One case of minor hypopigmentation was reported, but was resolved after one month.

\section{Conclusion}

Application of 694-nm wavelength fractional toning ruby laser for treating melasma on the malar area showed significant improvement in the MSS. Moreover, the treatment could be repeatedly applied, with low complication rate and significant patient satisfaction.

\section{Key words}

Melasma; Laser therapy; Ruby Lasers 


\section{INTRODUCTION}

Melasma is a symmetrical hyperpigmented patch with gray to brown color typically presenting on forehead, malar area and chin. This pigmented lesion is prevalent in women, especially in pregnant women, and for this reason it is also known as the mask of pregnancy. Taking oral contraceptives or receiving hormonal replacement therapy also increase the risk, therefore melasma is troublesome for women worldwide. There are various treatments for melasma currently including topical agents, peels and lasers, ${ }^{2,3}$ however for dermal histologic type melasma which is the most common type among 3 skin layer types ${ }^{4}$; epidermal, dermal and epidermal-dermal mixed, and mixed type, the 2 former kinds of treatment are not fully effective. Recently, the usefulness of laser treatment has been proven in the treatment of so called those 'refractory melasma', and a plenty of lasers such as neodymium (Nd):yttrium aluminum garnet (YAG), erbium $(E r): Y A G$, alexandrite, ruby lasers are on trial and studied clinically. In this study, the effectiveness of ruby fractional toning laser in melasma treatment is assessed.

\section{MATERIALS AND METHODS}

\section{Patients and data assessment}

From January 2018 to December 2019, a retrospective study was conducted on 40 melasma patients who had written well-informed consents at a single institute. The study was approved by the institutional review board (IRB) and the investigators conducted the study in compliance with the ethical guidelines of the Declaration of Helsinki. The collected data were subject's sex, age, laser protocol and photographic findings for progress. Subjects received total 8 times of treatment at intervals of 2 to 3 weeks. At each treatment, and one month after the end of treatment, they visited the outpatient clinic for evaluation of the improvement and being taken pictures. In the study, two independent investigators assessed photos and objectively evaluated the severity of melasma, with categorial scale; Melasma Severity Scale (MSS) (Table 1). In addition, sub-

Table 1. Melasma Severity Scale (MSS) used for the study

\begin{tabular}{cc}
\hline Scale & \multicolumn{1}{c}{ Hyperpigmentation Status } \\
\hline 0 & Cleared: color of melasma lesions approximately equivalent to \\
& $\begin{array}{c}\text { surrounding normal skin or with } \\
\text { minimal residual hyperpigmentation }\end{array}$ \\
1 & Mild: slightly darker than the surrounding normal skin \\
2 & Moderate: moderately darker than the surrounding normal skin \\
3 & Severe: markedly darker than the surrounding normal skin \\
\hline
\end{tabular}

jects performed self-assessment by looking at the mirrors and pictures, and a survey of satisfaction (score between 0 and 3 points) was conducted at each visit after the first treatment and one month after the treatment was ended (Table 2). Complications such as pain during procedure, hyper- or hypopigmentation were also assessed.

\section{Protocols}

The laser used for the study was a 694-nm wavelength fractional toning ruby laser (Melastar ${ }^{\circledR}$; Asclepion, Jena, Germany) with a 3-rod tip of $7.1 \mathrm{~mm} \times 7.1 \mathrm{~mm}$ spot size, and the parameter was set to $2.5-3.5 \mathrm{~J} / \mathrm{cm}^{2}$ fluence and 1.5-2.0 Hz frequency. Topical anesthesia was performed on the malar area one hour before laser treatment, and only sunscreen was applied after the procedure.

\section{RESULTS}

All subjects were asian female with type III Fitzpatrick skin type and mean age was 49.2 years. The mean MSS assessed by investigators at initial visit was 1.9 (Table 3). There was sight increase in MSS between the second and third visit but after the third visit, there was continuous decrease of MSS. Especially at the 8th visit and 1-month after the end of treatment, MSS significantly improved compared to the first visit (Table 4, Fig. 1, 2). Subjects were not satisfied until the 4 th visit, but after the 4 th treatment, the

Table 2. Subject satisfaction score

\begin{tabular}{cl}
\hline Score & \multicolumn{1}{c}{ Comments } \\
\hline 0 & No improvement in melasma \\
1 & Slight improvement in melasma but less visible \\
2 & Visible improvement in melasma \\
3 & Prominent improvement in melasma \\
\hline
\end{tabular}

Table 3. Characteristics of the subjects at the first visit

\begin{tabular}{|c|c|}
\hline Age $(\%)$ & Number of subjects \\
\hline Mean (yr) & 49.2 \\
\hline $21-30$ & $4(10 \%)$ \\
\hline $31-40$ & $6(15 \%)$ \\
\hline $41-50$ & $13(32.5 \%)$ \\
\hline $51-60$ & $11(27.5 \%)$ \\
\hline $61-70$ & $6(15 \%)$ \\
\hline \multicolumn{2}{|c|}{ MSS } \\
\hline Mean & 1.9 \\
\hline 0 & 0 \\
\hline 1 & 11 \\
\hline 2 & 22 \\
\hline 3 & 7 \\
\hline
\end{tabular}


subjects' satisfaction score increased significantly (Table 5). No subject reported pain during laser treatment and there was 1 case of minor hypopigmentation which occurred at 8th visit and resolved 2 months after the end of treatment.

\section{DISCUSSION}

Well-known risk factors for melasma are female sex, pregnancy, ultraviolet (UV) ray exposure, Fitzpatrick skin type III or above, hormone intake and thyroid dysfunction. ${ }^{5}$ There's also a study suggesting familial history of melasma. ${ }^{4}$ This multifactorial acquired disorder makes challenging recurrent and relapsing nature. The diversity of causes was followed by the development of various treatment methods such as hydroquinone, retinoic acid, azelaic acid, kojic acid, corticosteroid, chemical peels, $\mathrm{CO}_{2}$ laser, Er:YAG, Nd:YAG laser and Q-switched laser, etc. A specific location of the lesion or studies suggesting that symptoms aggravate after exposure to UV ray demonstrate that UV exposure is one of the most important causes of melasma

Table 4. MSS assessed by investigators at every visit time

\begin{tabular}{lcc}
\hline \multicolumn{1}{c}{ Visit time } & MSS & $p$-value \\
\hline 1st & 1.90 & \\
2nd & 1.83 & 0.54 \\
3rd & 1.88 & 0.16 \\
4th & 1.78 & 0.12 \\
5th & 1.73 & 0.09 \\
6th & 1.63 & 0.07 \\
7th & 1.53 & 0.05 \\
8th & 1.43 & 0.01 \\
1 month after the end of & 1.20 & $<0.001$ \\
treatment & & \\
\hline
\end{tabular}

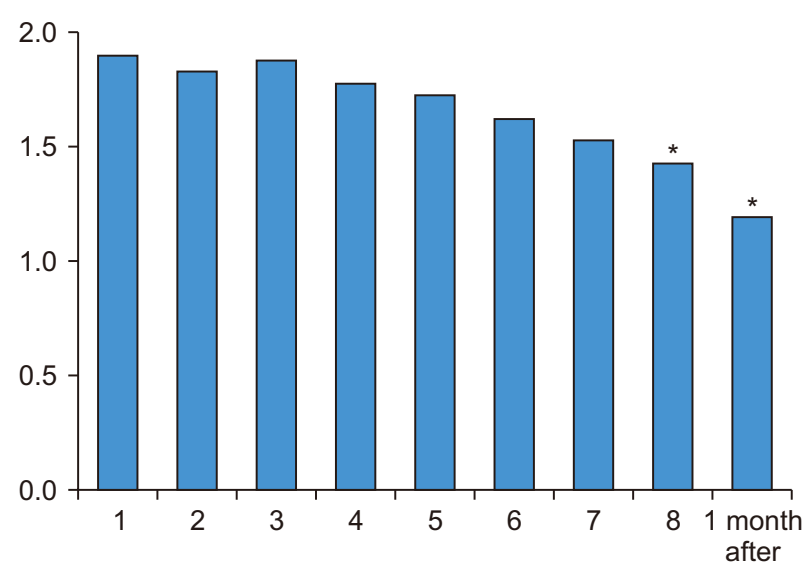

Fig. 1. Mean Melasma Severity Scale (MSS) at every visit. Highlighted bar with '*' is the value with statistical significance. The rightmost bar is 1 month after treatment. and support the usefulness of sunscreen.

Three pathologic types of melasma are generally known; Epidermal, dermal and mixed type. ${ }^{6}$ The latter 2 are more common types however topical agents which are the current first-line treatment for melasma, and chemical peels are partially effective on these two types.
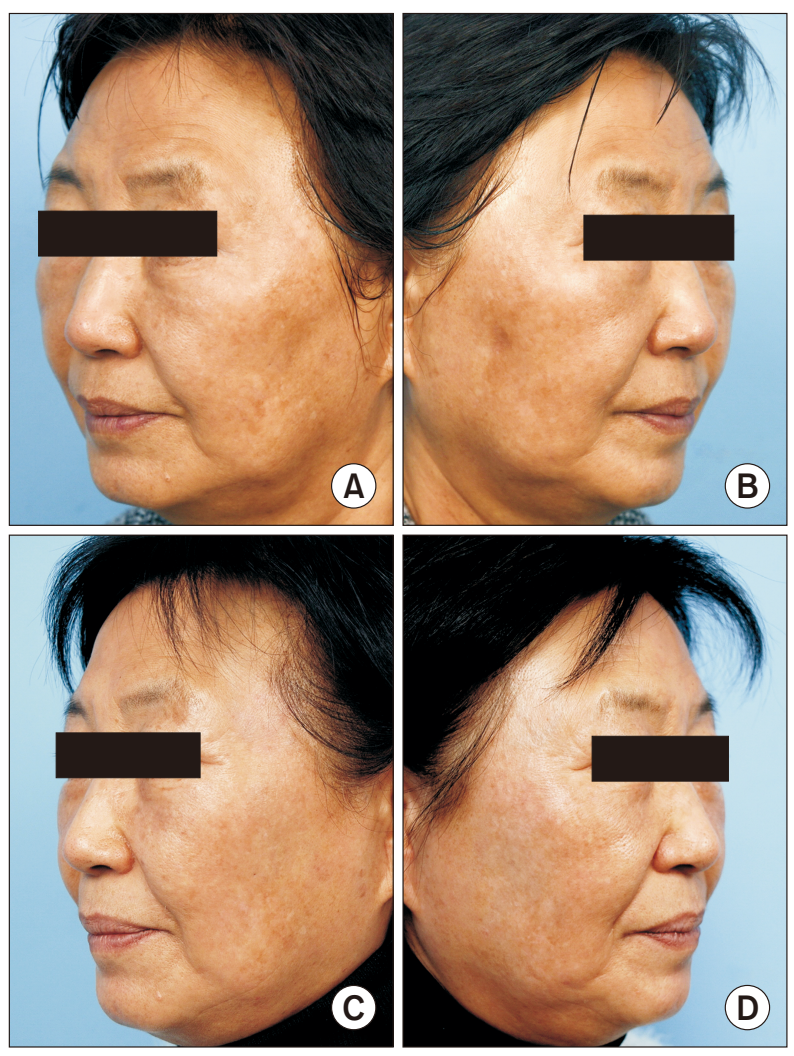

Fig. 2. Initial (A, B) and long-term follow up (C, D, 1 month after 8th visit) photographic findings of 41 year-old Korean female subject with Fitzpatrick skin type III. Her initial MSS is 2 (moderately darker than the surrounding normal skin). She reports 'score-3-satisfaction' after 8 times of 624-nm wave-length fractional toning ruby laser treatment.

Table 5. Subjects satisfaction score per visit. Score was significantly higher from the 5 th visit which means after 4 times of procedure

\begin{tabular}{lcc}
\hline Visit time & $\begin{array}{c}\text { Subjects } \\
\text { Satisfaction Score }\end{array}$ & $p$-value \\
\hline 2nd & 1.03 & \\
3rd & 1.08 & 0.35 \\
4th & 1.13 & 0.18 \\
5th & 1.48 & 0.003 \\
6th & 1.53 & 0.001 \\
7th & 1.63 & $<0.001$ \\
8th & 1.63 & $<0.001$ \\
1 month after the end of & 1.75 & $<0.001$ \\
treatment & & \\
\hline
\end{tabular}


The melanin pigments stored in the melanosome of the melanocyte, which are mainly present in the basal layer of the epidermis, are tansported through the dendrite to the keratinocyte which consists $90 \%$ of the epidermis.

Laser toning has toning effect by reducing the number of dedrites of melanin cells in which a lot of these melanosomes are distributed, reducing delivery to keratinocytes. ${ }^{7}$ The ruby laser characterized by 694-nm wavelength shows about 8.8 times higher absorption on menalin than the conventional 1,064-nm wavelength Nd:YAG laser or 755-nm wavelength alexandrite laser which are widely used in Asian countries, and lower absorption of oxyhemoglobin and water decreases thermal damage of blood vessels and adjacent tissues. High absorption rate by superficial melanin when using 694-nm wave length ruby laser, however, can lead to permanent hypopigmentation or depigmentation in darker-skinned individual such as Asian. However in this study, after using lowfluence and multi-pass laser toning, 1 minor hypopigmentation occurred after 8 times of laser treatment but it resolved 1 month after the end of treatment. Fractional laser separates tiny treatment zones and the adjacent tissue that is not irradiated, which can result in less irritation, inflammation and shorter healing time compared to non-fractional laser treatment. ${ }^{8}$

Since this study observed the progress of melasma of the subjects over about 2 years, photographing and efficacy assessment were performed not only in winter but also in summer. Education for subjects to minimize ultraviolet exposure was done in advance however, the exposure might be inevitable and differences in skin pigmentation according to seasons could not be excluded. In other words, if the treatment was performed in the summer and the final effect was evaluated in the winter, the result could be inaccurate due to natural skin lightening.

Investigating the satisfaction of subjects which can be altered by various external factors, is quite subjective and it should not be judged to have more than clinical meaning but just satisfaction itself. However, the satisfaction score showed a similar tendency to the objective indicator, MSS, which was evaluated by two independent investigators. In addition, considering the nature of melasma; high relapsing rate, the efficacy of 694-nm wavelength fractional toning laser through long term follow up should be examined.

\section{CONCLUSION}

Treatment using a 694-nm wavelength fractional toning ruby laser for melasma on the malar area showed improvement in MSS as the treatments was repeated, and patient satisfaction was also significant. It showed less complication including pain or post-inflammatory hypopigmentation compared to conventional laser treatment.

\section{CONFLICT OF INTEREST}

Eun Soo Park is an editorial board member of the journal but was not involved in the review process of this manuscript. Otherwise, there is no conflict of interest to declare.

\section{FUNDING}

This work was supported by the Soonchunhyang University Research Fund.

\section{REFERENCES}

1. Bolanca I, Bolanca Z, Kuna K, Vuković A, Tuckar N, Herman R, et al. Chloasma--the mask of pregnancy. Coll Antropol 2008;32 Suppl 2:139-41.

2. Del Rosario E, Florez-Pollack S, Zapata L Jr, Hernandez K, Tovar-Garza A, Rodrigues M, et al. Randomized, placebo-controlled, double-blind study of oral tranexamic acid in the treatment of moderate-to-severe melasma. J Am Acad Dermatol 2018;78:363-9.

3. Grimes PE, ljaz S, Nashawati R, Kwak D. New oral and topical approaches for the treatment of melasma. Int J Womens Dermatol 2018;5:30-6.

4. Achar A, Rathi SK. Melasma: a clinico-epidemiological study of 312 cases. Indian J Dermatol 2011;56:380-2.

5. Lee AY. An updated review of melasma pathogenesis. Dermatol Sinica 2014;32:233-9.

6. Kang WH, Yoon KH, Lee ES, Kim J, Lee KB, Yim H, et al. Melasma: histopathological characteristics in 56 Korean patients. Br J Dermatol 2002;146:228-37.

7. Mun JY, Jeong SY, Kim JH, Han SS, Kim IH. A low fluence Qswitched Nd:YAG laser modifies the 3D structure of melanocyte and ultrastructure of melanosome by subcellular-selective photothermolysis. J Electron Microsc (Tokyo) 2011;60:11-8.

8. Tierney EP, Hanke CW. Review of the literature: treatment of dyspigmentation with fractionated resurfacing. Dermatol Surg 2010;36:1499-508.

How to cite this article: Kim JH, Park ES, Nam SM, Cha HG. Efficacy of 694-nm fractional toning ruby laser in the treatment of malar melasma. Med Lasers 2021;10:45-48. https://doi.org/10.25289/ML.2021.10.1.45 